\title{
COMPLEMENTARY AND DUPLICATE GENE INTERACTIONS IN BIOMETRICAL GENETICS
}

\author{
KENNETH MATHER \\ University of Southampton
}

Received 28.v.66

\section{COMPLEMENTARY AND DUPLICATE RELATIONSHIP}

$T_{H E}$ phenotypic differences among the nine genotypes provided by the combinations of two pairs of alleles, $A-a$ and $B-b$, can be described completely in terms of eight quantities. Using the notation of Hayman and Mather (1955) there are:- $d_{a}$ and $d_{b}$, representing respectively the differences between the homozygotes $A A-a a$ and $B B-b b ; h_{a}$ and $h_{b}$, representing the effects of dominance at the two loci; $i_{a b}$ representing the interaction between the two $d$ 's; $j_{a / b}$ and $j_{b / a}$ representing the interactions between $d_{a}$ and $h_{b}$ and $d_{b}$ and $h_{a}$ respectively; and $l_{a b}$ measuring the interaction between the two $h$ 's. The phenotypes associated with the nine genotypes are set out in terms of these quantities in table $\mathrm{I}$.

TABLE I

The nine phenotypes associated with the nine genotypes produced by the combinations of two gene-pairs $\mathrm{A}-\mathrm{a}$ and $\mathrm{B}-\mathrm{b}$

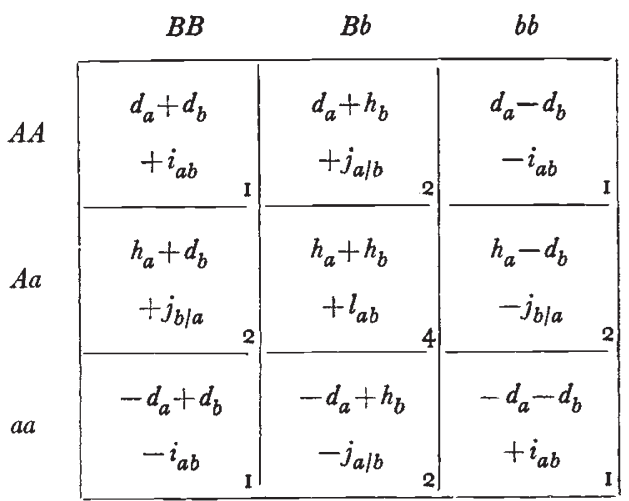

The numbers in the lower right corners of the nine cells of the table are the proportions expected for the nine gene combinations in an $F_{2}$.

Although the notation is that of Hayman and Mather, the two $j$ 's and $l$ are not defined in the way used by those authors, but follow the definitions whose use Van der Veen (1959) attributes to Smith and Robson, and which are more convenient for our present purpose. The interactions as defined in the two ways can always be converted into one another.

Each of the four interactions may, of course, be positive, negative or zero, as may the two $h$ 's. We can thus recognise $3^{6}=729$ types of relation between the two gene pairs in their expression. Many of 
these are merely mirror-images of one another, produced by simple changes in sign of the six quantities. Others are bizarre relationships of little but academic interest. Our present concern is with the two great classes of relationship that we may call complementary and duplicate gene interactions, after the classical types of interaction associated respectively with the $9: 7$ and I5 : I ratios in $F_{2}$ (Darlington and Mather, I949, fig. 38).

If we set $d_{a}=d_{b}=h_{a}=h_{b}=i_{a b}=j_{a \mid b}=j_{b \mid a}=l_{a b}$ we find that $A A B B, A a B B, A A B b$ and $A a B b$ have a common phenotype as do $a a B B$, $a a B b, A A b b, A a b b$ and $a a b b$, so giving the $9: 7 F_{2}$ ratio characteristic of the classical complementary gene relationship. Similarly putting $d_{a}=d_{b}=h_{a}=h_{b}=-i_{a b}=-j_{a / b}=-j_{b / a}=-l_{a b}$ makes all the phenotypes alike except that of $a a b b$, so giving the $1_{5}:$ I ratio of the classical duplicate gene relationship. These two situations are shown diagrammatically in the upper row of table 2. Each of these has a counterpart which is a mirror image in the sense that its tail points in the opposite direction, towards the lesser expression of the character instead of the greater expression and vice versa. This counterpart relation is obtained in each case by simultaneously changing the sign of the two $h$ 's, $i$ and $l$. They are illustrated in the lower row of table 2. Thus the characteristics of the complementary relationship are that the two $j$ 's are always $+\mathrm{ve}$, with the $h$ 's, $i$ and $l$ having the same sign as one another, all being $+v e$ for one mirror image and all - ve for the other. The characteristics of the duplicate relation are that the $j$ 's are always - ve, with the two $h$ 's having the same sign as one another but opposite to $i$ and $l$ which in turn are of like sign. The one mirror image is given by the $h$ 's being $+\mathrm{ve}$, with $i$ and $l-\mathrm{ve}$, while the other has $h$ 's - ve and $i$ and $l+\mathrm{ve}$.

Having established the characteristic sign of $h, i, j$ and $l$ for the two classes of relationship, we can generalise the relationships by letting these quantities take values less than $d$, when we may say we have a partial complementary or duplicate relationship, as the case may be, or even by letting the interaction quantities take values greater than $d$ so giving a super-complementary or super-duplicate relationship as the case may be. If $h<d$, to put $j=l=d$ would give a superinteractive relationship, and it is convenient to regard the full interaction as given in such a case by $j=\sqrt{d \bar{h}}$ and $l=h$ or $l_{a b}=\sqrt{h_{a} h_{b}}$ where $h_{a} \neq h_{b}$.

It is necessary for the classical complementary and duplicate relationships that $d_{a}=d_{b}$. If $d_{a} \neq d_{b}$, the complementary relationship degenerates into recessive epistasis, characterised by the $9: 3: 4$ ratio in $F_{2}$, with $d_{a}=h_{a}=j_{b / a}=i_{b a}$ and $d_{b}=h_{b}=j_{a \mid b}=l_{a b}$, or $d_{a}=-h_{a}=-i_{a b}=j_{b / a}$ and $d_{b}=-h_{b}=j_{a \mid b}=-l_{a b}$. Similarly, when $d_{a} \neq d_{b}$, the duplicate relationship degenerates into dominant epistasis characterised by the I 2:3: I ratio in $F_{2}$, with $d_{a}=h_{a}=-i_{a b}=-j_{b / a}$ and $d_{b}=h_{b}=-j_{a / b}=-l_{a b}$ or $d_{a}=-h_{a}=i_{a b}=-j_{b / a}$ and $d_{b}=-h_{b}=-j_{a / b}=l_{a b}$ 
TABLE 2

The complementary and duplicate relationships

Complementary
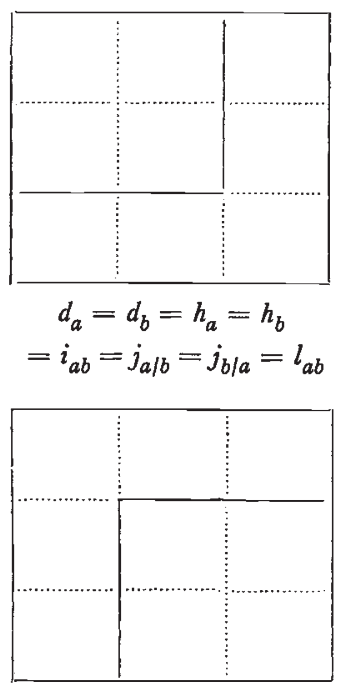

$d_{a}=d_{b}=-h_{a}=-h_{b}$

$=-i_{a b}=j_{a / b}=j_{b / a}=-l_{a b}$
Duplicate

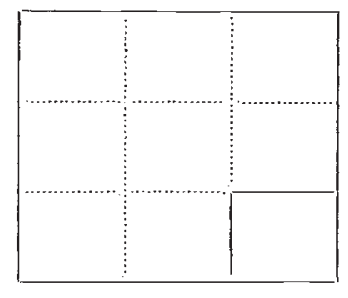

$d_{a}=d_{b}=h_{a}=h_{b}$

$=-i_{a b}=-j_{a / b}=-j_{b / a}=-l_{a b}$

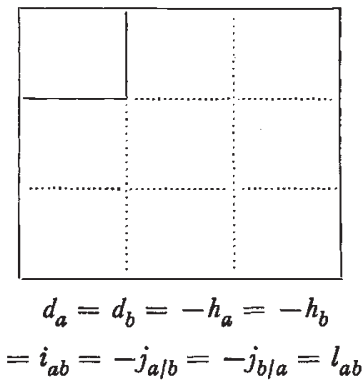

Signs of the eight quantities in the four relationships

\begin{tabular}{|c|c|c|c|c|c|c|}
\hline \multicolumn{2}{|c|}{ Quantity } & $d$ 's & $h$ 's & $i$ & $j$ 's & $l$ \\
\hline \multirow{3}{*}{\multicolumn{2}{|c|}{$\begin{array}{l}\text { Complementary-upper } \\
\text { - lower } \\
\text { Duplicate } \quad \text { - upper }\end{array}$}} & + & + & + & + & + \\
\hline & & + & - & - & + & - \\
\hline & & + & \pm & $\bar{t}$ & - & $\overrightarrow{+}$ \\
\hline
\end{tabular}

\section{THE VARIANCE OF $F_{2}$}

The mean expression of the character in an $F_{2}$ segregating for the two genes of table I departs from the mid-par ent value by $\frac{1}{2} h_{a}+\frac{1}{2} h_{b}+\frac{1}{4} l$. The variance of the expression of the character is

$$
\begin{aligned}
V_{1 F 2}=\frac{1}{2}\left(d_{a}-j_{a \mid b}\right)^{2}+\frac{1}{2}\left(d_{b}-j_{b \mid a}\right)^{2} & +\frac{1}{4}\left(h_{a}+\frac{1}{2} l_{a b}\right)^{2} \\
& +\frac{1}{4}\left(h_{b}+\frac{1}{2} l_{a b}\right)^{2}+\frac{1}{4} i_{a b}^{2}+\frac{1}{8}\left(j_{a \mid b}^{2}+j_{b \mid a}^{2}\right)+\frac{1}{16} l_{a b}^{2} .
\end{aligned}
$$

When $j_{a / b}$ and $j_{b / a}$ are positive $\left(d_{a}+\frac{1}{2} j_{a \mid b}\right)>d_{a}^{2}$ and $\left(d_{b}+\frac{1}{2} j_{b / a}\right)^{2}>d_{b}^{2}, V_{1 F 2}$ will then be greater than it would in the absence of these interactions. Similarly if $h_{a}, h_{b}$ and $l_{a b}$ are of the same sign $\left(h_{a}+\frac{1}{2} l_{a \mid b}\right)^{2}>h_{a}{ }^{2}$ and $\left(h_{b}+\frac{1}{2} l_{a b}\right)^{2}>h_{b}{ }^{2}$, the value of $V_{1 F 2}$ again being increased by the interaction. As we have seen, the characteristics of the complementary relationship are that the $j$ 's are + ve and the $h$ 's, $i$ and $l$ are of like sign to one another. Interactions of the complementary type must thus 
always increase the variance of an $F_{2}$. They will indeed increase the variance of any population in which the two gene-pairs are segregating independently of one another.

With the duplicate relationship on the other hand, the $j$ 's are -ve while $l$ is of the opposite sign to the $h$ 's. Such a relationship will therefore tend to reduce $V_{1 F 2}$ below the value it takes in the absence of interaction. It is not difficult to show that if $i$ is zero, the variance is at its minimum when $-j_{a / b}=d_{a},-j_{b / a}=d_{b}$, and $-l_{a b}=h_{a}=h_{b}$. With $i$ other than o, however, the minimum value of $V_{1 F 2}$ is attained when the $j$ 's are somewhat smaller than the $d$ 's and $l$ somewhat smaller than the $h$ 's. If we put $d_{a}=d_{b}=h_{a}=h_{b}$ and $i_{a b}=j_{a / b}=j_{b / a}=l_{a b}=-\theta d$, we find that $V_{1 F 2}$ is at a minimum when $\theta=0.8 . \quad V_{1 F 2}$ is rising again when the interaction is full, i.e. $\theta=\mathrm{I}$, and it rises further with a superinteraction, i.e. $\theta>\mathrm{I}$. It regains the value achieved in the absence of interaction when $\theta=\mathrm{I} \cdot 6$. Assuming that, apart from sign, all the $d$ 's and $h$ 's are of unit value the change of $V_{1 F 2}$ with $\theta$ is shown in fig. I.

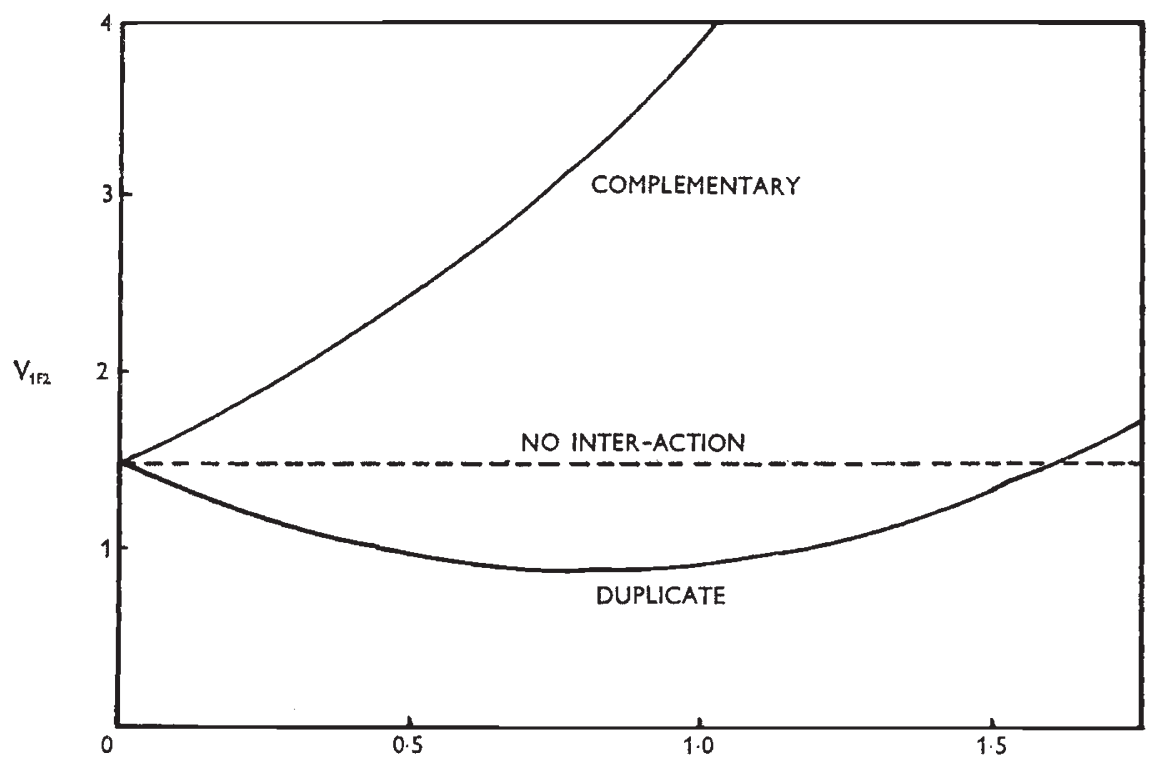

Fig. 1.-The effect on the variance of $F_{2}\left(V_{1 F 2}\right)$ of complementary and duplicate interactions. Two gene pairs are assumed with $d_{a}=d_{b}=h_{a}=h_{b}=\mathrm{I}$, and $i_{a b}=j_{a / b}$ $=j_{b / a}=l_{a b}=\theta d$, apart from necessary reversals of sign (see in the text). $\theta$ measures the intensity of the interaction. At $\theta=0$ interaction is absent. At $\theta=1$ interaction is complete and the two genes accord exactly with the classical complementary or duplicate pattern, as the case may be, in their interaction. $0<\theta>1$ implies partial interaction and $\mathrm{l}<\theta$, super-interaction.

Continuing selection towards a single optimum phenotype will be expected to favour reduction of variation. It will be expected therefore to favour duplicate types of interaction, and to do so particularly where selection is directional, though it should not, however, favour super-duplicate interaction $(\theta>I)$ or even necessarily favour complete interaction of this kind $(\theta=\mathrm{I})$. It is worth noting that there is 
evidence of the duplicate interaction thus expected in the genetical architecture of characters under continuing directional selection (Breese and Mather, I96o; Mather I966). Complementary interactions, on the other hand, raise the variance and so should never be favoured by continuing selection towards a single optimum. It is possible, however, that they will be favoured in some situations by disruptive selection towards two or more optima.

\section{DIALLEL CROSSES}

In diallel crosses where the alleles at each locus are equal in frequency and where there is no association among the genes at different loci, the $W_{r} / V_{r}$ graph will be a straight line in the absence of interactions among the non-allelic genes (Jinks, I954; Hayman, 1954). Interactions of the kind we have been discussing cause departures from this rectlinear relation of $W_{r}$ and $V_{r}$ and may be detected by these departures.

The departures from rectlinearity of the $W_{r} / V_{r}$ graph brought about by complementary and duplicate relationships are illustrated in fig. 2. It has been assumed in this as in fig. I that the $d$ 's and $h$ 's

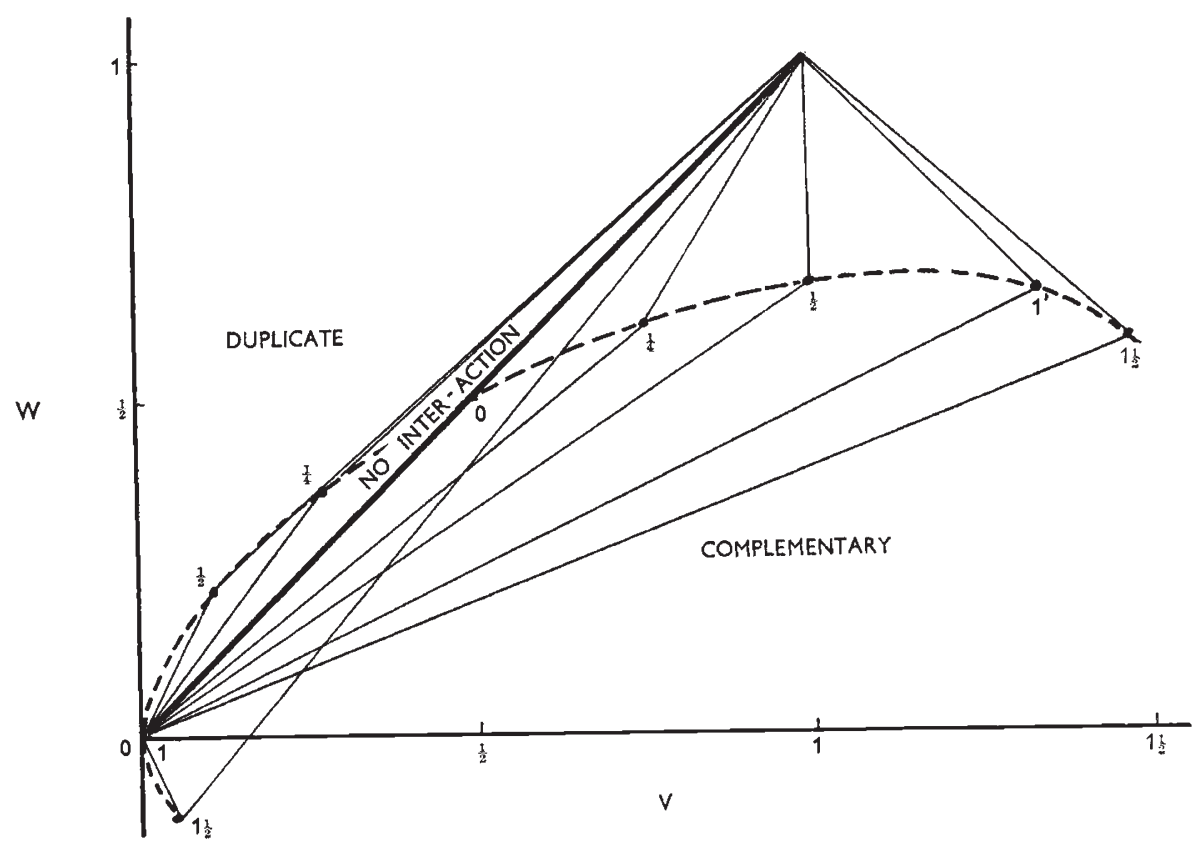

FIG. 2. - The effect of complementary and duplicate interactions between two gene pairs on the $W_{r} / V_{r}$ graph from a diallel cross. With no interaction $(\theta=0)$ the graph is a straight line with the middle point half way between the two extreme points. Complementary interaction results in this middle point moving to the right and upwards, and duplicate interaction results in it moving to the left and downwards. The $W_{r} / V_{r}$ graph thus ceases to be a straight line where interaction supervenes, being concave upwards with complementary and concave downwards with duplicate interaction. The dotted curve shows the path of the middle point as it moves under the influence of interaction, the numbers indicating the values of $\theta$ to which the points correspond.

G 2 
were all of equal magnitude, and also that $i=j=l$, apart from the necessary differences in sign. $\theta$ was given the value $\frac{1}{4}, \frac{1}{2}$, I and $\mathrm{I} \frac{1}{2}$ in turn in order to illustrate the progress of the departures from rectlinearity of $W_{r} / V_{r}$ as the strength of the interaction increased until it was first complete with $\theta=\mathrm{I}$ and then a super-interaction with $\theta=\mathrm{I} \frac{1}{2}$. The actual numerical values of $d_{a}$ etc. were, however, varied from case to case in order to keep constant the position of the rightmost point on the graph and so to bring out more clearly the progress of the departures from rectlinearity as $\theta$ changed. Thus with $\theta=\mathrm{I}$ and $d_{a}=\mathrm{I}$ the rightmost point of the graph falls at $W_{r}=V_{r}=3$, whether the interaction is complementary or duplicate and at $W_{r}=V_{r}=2$ when $\theta=0$. At $\theta=\frac{1}{2}$ and $d_{a}=\mathrm{I}$ it falls at $W_{r}=V_{r}=2 \frac{1}{4}$. In drawing fig. 2, therefore, the scale of the graph has been adjusted to make this point coincide with the corresponding point where $\theta=0$. The same has been done for $\theta=\frac{1}{4}$ and $\theta=\mathrm{I} \frac{1}{2}$.

Since $d_{a}=d_{b}, h_{a}=h_{b}$ and $j_{a \mid b}=j_{b \mid a}$ there are only three points on the $W_{r} / V$, line, because the centre two arrays of the diallel table, stemming from recurrent parents $A A b b$ and $a a B B$, give identical results. The leftmost point in all cases is at $(0,0)$ and, as already explained, the rightmost points have been made to coincide. The middle point falls on the straight line, mid-way between the end points, in the absence of interaction $(\theta=0)$. With a complementary relation between the genes this middle point falls below the line and moves to the right, the departure increasing as the strength of the interaction increases. Complementary interaction thus causes the $W_{v} / V_{r}$ line to be concave upwards, though when $\theta=I$ the departure is so great that the concavity of the line would be hard to discern if the results from lower values of $\theta$ were not available to reveal the true nature of the graph. Complementary interaction also causes the middle point to move towards the right end of the line.

Duplicate interactions have the opposite effect. The middle point is raised above the line and moved to the left. Thus with duplicate interaction the line is characteristically concave downwards and the middle point tends to cluster with the leftmost point. It will be observed that, for equal values of $\theta$, the concavity is much less with duplicate than with complementary interaction. Duplicate interaction might thus be expected to be more difficult to detect in diallel crosses than complementary, and Professor J. L. Jinks kindly allows me to say that empirically he has found this to be the case.

It should be noted that the direction (as distinct from the type) of the interaction has no effect on the $W_{r} / V_{r}$ graph: the complementary relation shown in the lower row of table I gives exactly the same $W_{r} / V_{r}$ points as its counterpart in the upper row of the table, and the same is true for the two duplicate relationships.

\section{SUMMARY}

The classical complementary and duplicate types of gene inter- 
action are defined in biometrical terms, and the definition is extended to cover incomplete and super-interactions of both types.

Complementary interactions increase the variance of segregating families and populations, but duplicate interactions generally decrease the variance. Thus selection towards a single optimum phenotype can favour duplicate but not complementary interactions. Complementary interactions could perhaps sometimes be favoured by disruptive selection towards two or more optima.

With diallel crosses, the $W_{r} / V_{r}$ graph becomes characteristically concave upwards with complementary, and concave downwards with duplicate interactions. Further, the points tend to cluster at the right or upper end of the line with the complementary, and at the left or lower end of the line with the duplicate relationship.

\section{REFERENCES}

BREESE, E. L., AND MATHER, K. rg6o. The organisation of polygenic activity within a chromosome in Drosophila. II. Viability. Heredity, 14, 375-399.

DARLington, G. D., AND MATHER, K. 1949. The Elements of Genetics. London, Allen and Unwin.

HAYMAN, B. I. I954. The theory and analysis of diallel crosses. Genetics, 39, 789-8o9. HAYMAN, B. I., AND MATHER, K. I955. The description of genic interaction in continuous variation. Biometrics, $I I, 69-82$.

JINKS, J. L. I954. The analysis of continuous variation in a diallel cross of Nicotiana rustica. Genetics, 39, 767-788.

mather, K. Ig66. Variability and selection. Proc. Roy. Soc. B, I64, 328-340.

VAN DER VEEN, J. H. 1959. Test of non-allelic interaction and linkage for quantitative characters in generations derived from two diploid pure lines. Genetica, 30, $201-232$. 